\title{
3 Research S Suare \\ Role of LRG1 in the Cell Proliferation, Migration, and Prognosis of Pancreatic Cancer
}

Jie Hua

Fudan University Shanghai Cancer Center

Qingcai Meng

Fudan University Shanghai Cancer Center

Chen Liang

Fudan University Shanghai Cancer Center

Miaoyan Wei

Fudan University Shanghai Cancer Center

Jiang Liu

Fudan University Shanghai Cancer Center

Bo Zhang

Fudan University Shanghai Cancer Center

\section{Wei Wang}

Fudan University Shanghai Cancer Center

Jin Xu

Fudan University Shanghai Cancer Center

Xianjun Yu ( $\nabla$ yuxianjun@fudanpci.org)

Fudan University Shanghai Cancer Center

Si Shi

Fudan University Shanghai Cancer Center

\section{Research}

Keywords: LRG1, DTX3L, pancreatic cancer, prognosis, proliferation, migration

Posted Date: July 23rd, 2021

DOI: https://doi.org/10.21203/rs.3.rs-733520/v1

License: (1) This work is licensed under a Creative Commons Attribution 4.0 International License.

Read Full License 


\section{Abstract}

Background: The aim of this study was to explore the role of leucine-rich a2-glycoprotein 1 (LRG1) in the biological function and prognosis of pancreatic cancer.

Methods: LRG1 was detected in serum and tissue specimens from patients with pancreatic cancer by enzyme-linked immunosorbent assay (ELISA), qRT-PCR, western blotting, and immunohistochemical (IHC) analysis. LRG1-overexpressing and LRG1-knockdown cell lines were established with lentiviral vectors containing LRG1-overexpression and shRNA plasmids, respectively. Colony formation, Cell Counting Kit-8 (CCK-8), wound healing, Transwell migration, and in vivo tumorigenicity assays were conducted to assess proliferation and migration of the pancreatic cancer cells. RNA sequencing was performed to identify potential downstream molecules of LRG1.

Results: Serum LRG1 levels were significantly elevated in patients with pancreatic cancer compared with healthy controls. The mRNA and protein levels of LRG1 were higher in cancer tissues than in adjacent normal tissues. High LRG1 expression was significantly associated with shorter overall survival and found to be an independent risk factor for poor prognosis. Additionally, LRG1 dramatically promoted cell proliferation and migration in vitro and accelerated tumor growth in vivo. By RNA sequencing, we identified Deltex (DTX)-3-like E3 ubiquitin ligase (DTX3L) as a potential downstream molecule of LRG1. Further validation experiments confirmed a positive correlation between LRG1 and DTX3L.

Conclusions: LRG1 is a valuable prognostic marker for pancreatic cancer that plays a crucial role in cell proliferation and migration. Targeting LRG1 or the downstream molecule DTX3L provides a novel strategy for the treatment of pancreatic cancer.

\section{Background}

Pancreatic cancer remains one of the most lethal malignant tumors. Although improvements in surgical techniques and chemotherapy regimens have produced survival benefits for patients with pancreatic cancer [1-3], the 5-year survival rate remains relatively low [4]. To date, except for olaparib, targeted therapies and immunotherapies have not yet made a major breakthrough in pancreatic cancer [5]. Therefore, there is an urgent need to unravel the mechanisms of pancreatic cancer development and progression and to identify potential therapeutic targets.

Leucine-rich a2-glycoprotein 1 (LRG1), a member of the leucine-rich-repeat protein superfamily, was first identified as an inflammatory protein in human serum by Haupt and Baudner [6]. It was not until the last decade that the biological function of LRG1 in cancer was recognized. In colorectal cancer, LRG1 was found to be remarkably elevated and mediate epithelial-to-mesenchymal transition and angiogenesis through HIF-1 a activation [7]. LRG1 was also found to be a promising diagnostic marker for non-smallcell lung cancer [8, 9], biliary tract cancer [10], and ovarian cancer [11]. In addition, the LRG1 level is an independent prognostic factor for gastric cancer, and inhibition of LRG1 suppressed the growth of gastric cancer cells in vitro [12]. Previous studies have also reported the serum LRG1 level as an important 
biomarker for the early detection of pancreatic cancer [13] and a correlation between an increased LRG1 level and progressive clinical stages [14]. However, the prognostic effect of LRG1 in a large cohort of pancreatic cancer patients remains unclear. The role of LRG1 in the biological behavior of human pancreatic cancer cells as well as its underlying mechanisms deserve further study.

In this study, we found that LRG1 was elevated in patients with pancreatic ductal adenocarcinoma (PDAC) and that high LRG1 expression predicted a poorer prognosis than low LRG1 expression. LRG1 was also found to promote cell proliferation and migration in human pancreatic cancer cell lines. A remarkable correlation between LRG1 and its downstream molecule Deltex (DTX)-3-like E3 ubiquitin ligase (DTX3L) was identified, and DTX3L may serve as a potential target protein in future research.

\section{Materials And Methods Specimens and cell culture}

Serum samples from 100 pancreatic cancer patients who underwent surgical resection at the Fudan University Shanghai Cancer Center (FUSCC) from July 2012 to April 2016 were collected for the detection of serum LRG1 levels. Serum samples from 87 normal human subjects collected within the same period were used as controls. Forty pairs of frozen pancreatic cancer and adjacent normal pancreas tissue samples collected between March 2015 and June 2015 were retracted for tissue RNA or tissue protein extraction. Formalin-fixed, paraffin-embedded (FFPE) tissue specimens, including tissue microarrays (TMAs), were obtained for immunohistochemical (IHC) analysis. The study was approved by the Institutional Review Board of FUSCC, and written informed consent was obtained from the patients.

Human pancreatic cancer cell lines (MIA PaCa-2, PANC-1, BxPC-3, Capan-1, CFPAC-1, SW 1990, and HPAFII) were purchased from the American Type Culture Collection (ATCC) and cultured at $37^{\circ} \mathrm{C}$ in a humidified atmosphere containing $5 \% \mathrm{CO}_{2}$. A human pancreatic ductal epithelial cell line (HPDE) was a kind gift from Professor Ming-Sound Tsao (University Health Network, Toronto, Canada).

\section{Enzyme-linked Immunosorbent Assay (Elisa)}

Serum LRG1 levels were determined using a human LRG assay kit (IBL, Fujioka, Japan) according to the manufacturer's instructions. Each sample was assayed in triplicate. The absorbance at a wavelength of $450 \mathrm{~nm}$ was measured with an ELx800 absorbance microplate reader (BioTek Instruments, Winooski, VT, USA), and the serum LRG1 concentration was determined from a standard curve generated using internal control samples.

\section{Rna Isolation And Quantitative Rt-pcr (Qrt-pcr) Analysis}


Total RNA was isolated from frozen pancreatic tissues and cultured cells using RNAiso Plus reagent (Takara Bio Inc., Otsu, Shiga, Japan) according to the manufacturer's instructions. Reverse transcription was conducted using the PrimeScript RT reagent kit (Takara Bio Inc.). qRT-PCR was performed using the QuantiTect SYBR Green RT-PCR kit (QIAGEN, Hilden, NRW, Germany) on a QuantStudio 7 Flex system (Applied Biosystems, Foster City, MA, USA) in triplicate. The following primers were used:

\section{LRG1}

forward, 5'-CAGACAGCGACCAAAAAGC-3'

reverse, 5'-GGAACACCTGGCAGTCTTTG-3'

\section{DTX3L}

forward, 5'-CCAGGTTATGAGTCCTTTGGCAC-3'

reverse, 5'-TGCAGTTCGCTGTATTCCAGGG-3'

$\beta$-actin: forward, 5'-CTACGTCGCCCTGGACTTCGAGC-3'

reverse, 5'-GATGGAGCCGCCGATCCACACGG-3'

Relative LRG1 or DTX3L expression was determined in accordance with the $2^{-\Delta \Delta C t}$ method using $\beta$-actin as the reference gene.

\section{Western Blot Analysis}

Total protein extraction from frozen tissues and cultured cells and western blotting were performed as previously described [15]. The primary antibodies used were mouse anti- $\beta$-actin (66009-1-lg; Proteintech Group Inc, Rosemont, IL, USA), rabbit anti-LRG1 (HPA001888; Sigma-Aldrich, St. Louis, MO, USA) and rabbit anti-DTX3L (HPA010570; Sigma-Aldrich). $\beta$-Actin was used as a loading control. The immune complexes were detected by chemiluminescence (Immobilon; Millipore, Billerica, MA, USA). The intensity of the protein bands was quantified by ImageJ software (National Institutes of Health, Bethesda, MD, USA) and normalized to the $\beta$-actin protein levels.

\section{Ihc Assay}

FFPE specimens and TMAs were obtained from patients who underwent surgical resection at the FUSCC between March 2010 and April 2016 (last follow-up: July 2017). Briefly, heat-mediated antigen retrieval was performed using sodium citrate buffer ( $\mathrm{pH} \mathrm{6.0)}$ in a pressure cooker for $10 \mathrm{~min}$, and endogenous peroxidase activity was suppressed by incubation with a $3 \%$ solution of $\mathrm{H}_{2} \mathrm{O}_{2}$ for $15 \mathrm{~min}$. Slides were incubated with anti-LRG1 (1:200) or anti-DTX3L (1:200) antibodies overnight at $4^{\circ} \mathrm{C}$, followed by 
incubation with horseradish peroxidase polymer-conjugated secondary antibody. Based on staining intensity (H-scores: 0 , no staining [no coloration]; 1, weak staining [pale yellow]; 2, moderate staining [yellow]; 3 , strong staining [brown]) the samples were divided into two groups (low expression: 0 or 1 ; high expression: 2 or 3 ).

\section{Establishment Of Panc-1-Irg1-overexpressing And Mia Paca-2-Irg1-knockdown Cell Lines}

shRNA sequences were cloned into the hU6-MCS-Ubiquitin-EGFP-IRES-puromycin vector (GV248;

GeneChem, Shanghai, China) according to the manufacturer's instructions. The scramble shRNA and two LRG1-targeting shRNA sequences were used in this study: scramble shRNA, 5'-

TTCTCCGAACGTGTCACGT-3'; shLRG1-A, 5'-AGGCAACAAATTGCAAGTA-3'; shLRG1-B, 5'-

ATGTTTCTAGAACTCTGTT-3'. Lentiviral shRNA particles were generated by cotransfection of the lentiviral constructs with psPAX2 and pMD2.G into HEK-293T cells using Lipofectamine 3000 (Thermo Fisher Scientific, Rochester, NY, USA). To establish a stable LRG1-knockdown cell line, MIA PaCa-2 cells were infected by exposure to the lentiviral supernatant and polybrene $(2 \mu \mathrm{g} / \mathrm{ml})$ for 2 days. Infected cells were selected in culture medium containing puromycin $(1 \mu \mathrm{g} / \mathrm{ml})$.

The Flag-tagged coding sequences of human LRG1 were cloned into the BamH and Agel sites of the expression vector GV 492 (GeneChem). The preparation of lentiviral overexpression particles and cell infection were carried out as described above, and the empty GV 492 vector was used as a control.

\section{Rna Sequencing}

Total RNA was isolated from scrambled-MIA PaCa-2, shLRG1-A-MIA PaCa-2, shLRG1-B-MIA PaCa-2, LRG1-OE-PANC-1, and GV492-OE-PANC-1 cells using RNAiso Plus reagent (Takara Bio Inc.). RNA sequencing was performed using the Illumina HiSeq 4000 sequencing system.

\section{Colony Formation Assay}

Cells were seeded at an initial density of 1,000 cells/well in six-well plates and cultured for 2 weeks. Then, the cells were fixed with $4 \%$ paraformaldehyde (Sigma-Aldrich) for $30 \mathrm{~min}$ and stained with $0.1 \%$ crystal violet (Sigma-Aldrich) for $20 \mathrm{~min}$. Colonies containing more than 50 cells were counted under a light microscope.

\section{Cell Proliferation Assay}

Cells were seeded in 96-well plates at a density of $2 \times 10^{3}$ cells/well. Cell viability was quantified every 24 h using the Cell Counting Kit-8 (CCK-8; Dojindo, Kumamoto, Japan) assay. 


\section{Wound Healing Assay}

A wound healing assay was performed as previously described [7]. Briefly, when the confluency of the cells was ideal, a scratch wound was made across the center of the well with a pipette tip. The cells were then cultured in medium supplemented with $0.5 \%$ fetal bovine serum (FBS; Gibco, Carlsbad, CA, USA) for $24 \mathrm{~h}$ to allow wound closure. The degree of cell migration was analyzed as a percentage of wound area with ImageJ software.

\section{Transwell Migration Assay}

Twenty-four-well plates with 8- $\mu$ m pore filters (Corning Incorporated, Corning, NY, USA) were used for the cell migration assay. A total of $4 \times 10^{4}$ cells were seeded in the upper chamber of a Transwell plate and cultured in serum-free medium $(200 \mu \mathrm{l})$. The cells were allowed to migrate into the lower chamber, which was filled with culture medium $(800 \mu \mathrm{l})$ containing $10 \% \mathrm{FBS}$. After $48 \mathrm{~h}$ of incubation, the noninvaded cells that remained in the upper chamber were removed with cotton swabs. The migrated cells on the bottom surface of the membrane were fixed with $4 \%$ paraformaldehyde (Sigma-Aldrich), stained with $0.1 \%$ crystal violet (Sigma-Aldrich), and counted in five random microscopic visual fields.

In vivo tumorigenicity assay

Four- to six-week-old female BALB/c nude mice were purchased from the Shanghai Laboratory Animal Company (SLAC, Shanghai, China). Approximately $3 \times 10^{6}$ cells (in $50 \%$ Matrigel) were subcutaneously implanted into the second mammary fat pads of the mice ( $n=5$ for each cell line). After 4 weeks, the mice were euthanized, and the tumor specimens were surgically dissected and fixed with $4 \%$ paraformaldehyde. Xenograft tumor experiments were performed at the Laboratory Animal Center of Fudan University (Shanghai, China) according to an animal protocol approved by the Committee on the Ethics of Animal Experiments of Fudan University.

\section{Immunofluorescence Assay}

Immunofluorescence assays of cultured cells were performed on glass-bottom dishes (Thermo Fisher Scientific). Cells were fixed with $4 \%$ paraformaldehyde (Sigma-Aldrich), permeabilized with $0.5 \%$ Triton X100 (Sigma-Aldrich), blocked with bovine serum albumin (BSA; Sigma-Aldrich), and incubated with antiLRG1 or anti-DTX3L overnight at $4^{\circ} \mathrm{C}$. The cells were then incubated with fluorochrome-conjugated secondary antibody in the dark and photographed using a Leica TCS SP5 confocal laser scanning microscope. Double immunofluorescent staining of FFPE tissue specimens was performed as previously described [16].

\section{Statistical Analyses}


Statistical analyses were carried out with SPSS software (IBM, Chicago, IL, USA). Quantitative data were compared between every two groups using Student's $t$ test or the paired $t$ test. The Kaplan-Meier method was used to estimate overall survival, and comparisons between curves were performed using the logrank test. The Cox proportional hazard regression model was used to estimate the hazard ratio with a $95 \%$ confidence interval for variables associated with overall survival. Correlation analyses were performed using Spearman's correlation test. $P<0.05$ was used to indicate statistical significance.

\section{Results}

\section{LRG1 is upregulated in PDAC, and high LRG1 expression predicts poor prognosis}

To explore the differences in serum LRG1 levels between patients with PDAC and healthy controls, we performed ELISA on serum samples from 100 patients with PDAC and 87 healthy controls. We found that patients with PDAC had significantly elevated serum LRG1 levels compared with those of healthy controls ( $P<0.001$; Fig. 1a). A cut-point of $44.5 \mu \mathrm{g} / \mathrm{mL}$ was identified using X-tile software to differentiate the overall survival of patients with PDAC (Fig. 1b), with serum LRG1 levels higher than $44.5 \mu \mathrm{g} / \mathrm{mL}$ indicating poorer survival (Fig. 1C). The mRNA expression of LRG1 was measured in 28 paired samples of PDAC and adjacent normal tissues. As shown in Fig. 1d, the mRNA expression of LRG1 was significantly increased in the PDAC tissues. In 5 of 6 paired tissues, western blot analysis revealed that the protein level of LRG1 was higher in PDAC tissues than in adjacent normal tissues (Fig. 1e). To further confirm these findings, LRG1 protein expression in 35 paired tissue samples was determined by IHC analysis. According to $\mathrm{H}$-score analysis of the IHC-stained samples, the LRG1 protein was detected at a higher level in PDAC tissues than in adjacent normal tissues (Fig. 1f \& g). Finally, LRG1 protein expression was higher in all 7 tested pancreatic cancer cell lines than in normal HPDE cells (Fig. $1 \mathrm{~h}$ ).

To further explore the association between LRG1 expression and survival in patients with PDAC, we performed IHC analysis of TMAs containing 300 PDAC cases. The H-scores of samples from a total of 273 PDAC cases on the TMAs were analyzable, and among these cases, 92 showed low LRG1 expression, while 181 showed high LRG1 expression (Fig. 2a). Kaplan-Meier survival curves revealed that compared with low LRG1 expression, high LRG1 expression was associated with significantly poorer overall survival ( $P=0.010$; Fig. 2b). Further in silico analysis of 176 PDAC cases from The Cancer Genome Atlas (TCGA) dataset confirmed these findings, with a 5 -year survival rate of $70 \%$ in the low LRG1 group and $14 \%$ in the high LRG1 group ( $P=0.0026$; Fig. 2 c). Moreover, we performed Cox regression analysis with our 273 PDAC cases. After adjusting for the clinicopathological characteristics tumor stage, nodal status, tumor grade, and CA19-9 levels, high LRG1 expression was an independent risk factor for poor prognosis in PDAC patients (Fig. 2d). 


\section{Lrg1 Promotes The Growth And Migration Of Pancreatic Cancer}

To reveal the functional role of LRG1 in pancreatic cancer, we created the LRG1-overexpressing PANC-1 cell line and LRG1-knockdown MIA PaCa-2 cell line (Fig. 3a). To assess the effect of LRG1 on cell growth, we compared the growth rate of LRG1-overexpressing PANC-1 and LRG1-knockdown MIA PaCa-2 cells with the corresponding parental cells. LRG1 overexpression significantly promoted the growth of PANC-1 cells, while LRG1 knockdown dramatically inhibited the growth of MIA PaCa-2 cells (Fig. 3b). Further colony formation assays also demonstrated that LRG1 overexpression promoted cell proliferation, while LRG1 knockdown restrained proliferation (Fig. 3c). To further determine the effect of LRG1 on tumorigenicity and proliferation in vivo, we conducted a xenograft tumor experiment in nude mice. As shown in Fig. 3d, we found that tumor size was significantly larger in the LRG1-overexpressing PANC-1 group than in the control group $(P=0.0219)$. In the LRG1-knockdown MIA PaCa-2 group, the tumor size was much smaller than that in the scrambled group $(P<0.0001)$.

To determine the impact of LRG1 on cell migration, we performed wound healing and Transwell migration assays. LRG1 knockdown markedly decreased the motility of MIA PaCa-2 cells, as shown by a decrease in wound healing and reduction in migrated cells (Fig. 4a \& b). In contrast, LRG1 overexpression profoundly promoted cell migration by accelerating wound healing and increasing the number of migrated cells. Taken together, these findings suggest that LRG1 promotes the proliferation and migration of pancreatic cancer.

\section{Lrg1 Regulates The Downstream Molecule Dtx3I}

We next sought to identify molecular regulators that are involved in LRG1 signaling in pancreatic cancer. By RNA sequencing, we investigated differentially expressed genes after LRG1 overexpression/knockdown and identified DTX3L as a potential downstream molecule of LRG1. To further validate this hypothesis, we measured the mRNA levels of DTX3L after LRG1 overexpression or knockdown. We found that the mRNA levels of DTX3L were markedly decreased in LRG1-knockdown MIA PaCa-2 cells generated with either shRNA, while in LRG1-overexpressing PANC-1 cells, the mRNA levels of DTX3L were significantly elevated (Fig. 5a). In line with the qRT-PCR data, the impact of LRG1 overexpression or knockdown on the DTX3L protein level was confirmed by western blot analysis (Fig. 5b) and immunofluorescence assay (Fig. 5c).

To further reveal the potential correlation between LRG1 and DTX3L in pancreatic cancer specimens, we examined the expression of both LRG1 and DTX3L in TMAs (Fig. 6a). A positive correlation between LRG1 and DTX3L was observed, as shown by the H-scores (Spearman's $\mathrm{R}=0.148 ; P=0.0284 ; \mathrm{Fig} .6 \mathrm{~b}$ ). Moreover, qRT-PCR experiments showed that the mRNA level of DTX3L was correlated with that of LRG1 in 33 frozen pancreatic cancer tissues (Spearman's $\mathrm{R}=0.340 ; P=0.0277 ; \mathrm{Fig} .6 \mathrm{c}$ ). The TCGA data also confirmed our findings, showing a positive correlation between LRG1 and DTX3L expression (Spearman's 
$\mathrm{R}=0.180 ; P=0.015 ;$ Fig. $6 \mathrm{~d} \& \mathrm{e}$ ). Further double immunofluorescence staining demonstrated the coexpression of LRG1 and DTX3L in cancerous epithelial cells (Fig. 6f).

\section{Discussion}

In this study, we found that LRG1 was aberrantly overexpressed in the serum and cancer tissues of patients with PDAC. We further demonstrated that high LRG1 expression is an independent risk factor for poor prognosis. In addition, LRG1 plays a crucial role in the malignant characteristics of pancreatic cancer cells, including their proliferation, migration, and in vivo tumorigenicity. Moreover, LRG1 was significantly positively correlated with DTX3L, suggesting oncogenic signaling between LRG1 and DTX3L.

LRG1, a secreted glycoprotein, was found to be upregulated during granulocyte differentiation in earlier studies [17]. Furthermore, LRG1 is involved in the inflammatory process in several diseases, such as ulcerative colitis, rheumatoid arthritis, and appendicitis [18-21]. Over the past ten years, serum LRG1 has been found to be elevated in a variety of cancers. Early reports revealed that the combination of LRG1 and CA125 improved the early detection rate in ovarian cancer [22]. Similarly, serum LRG1 levels were higher in patients with non-small-cell lung cancer [9], gastric cancer [13], and colorectal cancer [23] than in healthy controls, indicating that LRG1 may be a broad-spectrum biomarker. A previously study also showed increased serum LRG1 levels in patients with PDAC compared with healthy controls [12], which was confirmed in our current study. Additionally, LRG1 was shown to have complementary benefits in capturing additional pancreatic cancer cases that were missed with the use of CA19-9 alone [13].

The abnormal expression of LRG1 in tumor tissues also has prognostic value in various cancers. A previous study revealed that high LRG1 expression is a statistically significant independent prognostic factor for poor overall survival in gastric cancer [13]. In esophageal squamous cell carcinoma, LRG1 was also confirmed to be an independent poor prognostic indicator for clinical outcomes [24]. In addition, LRG1 mRNA expression in breast cancer was associated with overall survival and progression-free survival but unrelated to the luminal subtype [25]. In this study, we demonstrated that high LRG1 expression correlated with poor overall survival and was an independent risk factor for poor prognosis in PDAC patients after adjusting for tumor stage, nodal status, tumor grade, and CA19-9 levels. These findings suggest that we may add LRG1 to the IHC panel of surgical specimens to facilitate prognostic evaluation.

Malignant characteristics, including abnormal proliferation and migration, are the basis of pancreatic cancer development and progression. In this study, we found that LRG1 overexpression promoted cell proliferation, while LRG1 knockdown suppressed proliferation. Moreover, LRG1 overexpression profoundly promoted cell migration, while LRG1 knockdown decreased motility. These findings are in line with previous reports showing the ability of LRG1 to promote tumors in other malignancies, such as gastric cancer [13], colorectal cancer [7], and glioblastoma [26]. Therefore, a strategy that targets LRG1 may inhibit the growth and migration of PDAC cells, which would hopefully improve outcome. 
A previous landmark study found that LRG1 promotes neovascularization by modulating TGF- $\beta$ signaling [27]. Another study showed that LRG1 can induce epithelial-to-mesenchymal transition and promote angiogenesis through HIF-1a activation [7]. In our study, we identified DTX3L, which is associated with the ADP-ribosyltransferase PARP9, as a downstream molecule of LRG1. We also demonstrated a positive correlation between LRG1 and DTX3L, indicating that DTX3L is involved in LRG1 signaling. Relevant experiments are ongoing, and both LRG1 and DTX3L are potential therapeutic targets.

\section{Conclusions}

In summary, LRG1 was significantly elevated in the serum and tissue of patients with PDAC, and high LRG1 expression is a promising prognostic marker for overall survival. LRG1 markedly promoted the proliferation and migration of pancreatic cancer cells and was positively correlated with DTX3L.

Targeting LRG1 or the downstream molecule DTX3L may provide a novel strategy for the treatment of pancreatic cancer.

\section{Abbreviations}

ATCC: American Type Culture Collection

BSA: bovine serum albumin

CCK-8: Cell Counting Kit-8

DTX3L: deltex (DTX)-3-like E3 ubiquitin ligase

ELISA: enzyme-linked immunosorbent assay

FFPE: formalin-fixed, paraffin-embedded

FUSCC: Fudan University Shanghai Cancer Center

HPDE: human pancreatic ductal epithelial

IHC: immunohistochemical

LRG1: leucine-rich a2-glycoprotein 1

PDAC: pancreatic ductal adenocarcinoma

qRT-PCR: quantitative Reverse Transcription-Polymerase Chain Reaction

SLAC: Shanghai Laboratory Animal Company

TCGA: The Cancer Genome Atlas 
TMAs: tissue microarrays

\section{Declarations}

\section{Ethics approval and consent to participate}

The study was approved by the Institutional Review Board of FUSCC, and written informed consent was obtained from the patients. Xenograft tumor experiments were performed at the Laboratory Animal Center of Fudan University (Shanghai, China) according to an animal protocol approved by the Committee on the Ethics of Animal Experiments of Fudan University.

\section{Consent for publication}

Not applicable.

\section{Availability of data and materials}

The data and materials are available form the corresponding author on reasonable request.

\section{Competing interests}

The authors declare that they have no competing interests.

\section{Funding}

This study was jointly funded by the National Natural Science Foundation of China (Nos. 81772555, 81802352 and 81902428), National Science Foundation for Distinguished Young Scholars of China (No. 81625016), Shanghai Sailing Program (Nos. 19YF1409400 and 20YF1409000), Shanghai Rising-Star Program (No. 20QA1402100), Shanghai Anticancer Association Young Eagle Program (No. SACACY19A06), Clinical and Scientific Innovation Project of Shanghai Hospital Development Center (Nos. SHDC12018109 and SHDC12019109) and Scientific Innovation Project of Shanghai Education Committee (No. 2019-01-07-00-07-E00057).

\section{Authors' contributions}

Hua J, Yu X, and Shi S conceived and designed the study; Hua J, Meng Q, and Liang C performed experiments, acquired and analyzed the data; Hua J, Wei M, and Liu J performed statistical analysis; Hua $J$, Zhang B, and Wang $W$ drafted the manuscript; $X u$ J, Yu X, and Shi S critically revised the manuscript; $\mathrm{Xu} J$, Yu X, and Shi S provided funding support; Xu J, Yu X, and Shi S supervised the study.

\section{Acknowledgements}

We thank Professor Yi Qin (Cancer Research Institute, Fudan University) for providing critical comments. 


\section{References}

1. Wray CJ, Ahmad SA, Matthews JB, Lowy AM. Surgery for pancreatic cancer: recent controversies and current practice. Gastroenterology. 2005;128:1626-41.

2. Conroy T, Desseigne F, Ychou M, Bouché O, Guimbaud R, Bécouarn Y, et al. FOLFIRINOX versus gemcitabine for metastatic pancreatic cancer. N Engl J Med. 2011;364:1817-25.

3. Von Hoff DD, Ervin T, Arena FP, Chiorean EG, Infante J, Moore M, et al. Increased survival in pancreatic cancer with nab-paclitaxel plus gemcitabine. N Engl J Med. 2013;369:1691-703.

4. Siegel RL, Miller KD, Fuchs HE, Jemal A. Cancer Statistics, 2021. CA Cancer J Clin. 2021;71:7-33.

5. Golan T, Hammel P, Reni M, Van Cutsem E, Macarulla T, Hall MJ, et al. Maintenance Olaparib for Germline BRCA-Mutated Metastatic Pancreatic Cancer. N Engl J Med. 2019;381:317-27.

6. Haupt $\mathrm{H}$, Baudner S. Isolation and characterization of an unknown, leucine-rich 3.1-S-alpha2glycoprotein from human serum. Hoppe Seylers Z Physiol Chem. 1977;358:639-46.

7. Zhang J, Zhu L, Fang J, Ge Z, Li X. LRG1 modulates epithelial-mesenchymal transition and angiogenesis in colorectal cancer via HIF-1a activation. J Exp Clin Cancer Res. 2016;35:29.

8. Li Y, Zhang Y, Qiu F, Qiu Z. Proteomic identification of exosomal LRG1: a potential urinary biomarker for detecting NSCLC. Electrophoresis. 2011;32: 1976-83.

9. Liu YS, Luo XY, Li QR, Li H, Li C, Ni H, et al. Shotgun and targeted proteomics reveal that pre-surgery serum levels of LRG1, SAA, and C4BP may refine prognosis of resected squamous cell lung cancer. $J$ Mol Cell Biol. 2012;4:344-7.

10. Sandanayake NS, Sinclair J, Andreola F, Chapman MH, Xue A, Webster GJ, et al. A combination of serum leucine-rich a-2-glycoprotein 1, CA19-9 and interleukin-6 differentiate biliary tract cancer from benign biliary strictures. $\mathrm{Br} \mathrm{J}$ Cancer. 2011;105:1370-8.

11. Wu J, Yin H, Zhu J, Buckanovich RJ, Thorpe JD, Dai J, et al. Validation of LRG1 as a potential biomarker for detection of epithelial ovarian cancer by a blinded study. PLoS One. 2015;10:e0121112.

12. Yamamoto M, Takahashi T, Serada S, Sugase T, Tanaka K, Miyazaki Y, et al. Overexpression of leucine-rich a2-glycoprotein-1 is a prognostic marker and enhances tumor migration in gastric cancer. Cancer Sci. 2017;108:2052-60.

13. Capello M, Bantis LE, Scelo G, Zhao Y, Li P, Dhillon DS, et al. Sequential Validation of Blood-Based Protein Biomarker Candidates for Early-Stage Pancreatic Cancer. J Natl Cancer Inst. 2017;109:djw266.

14. Furukawa K, Kawamoto K, Eguchi H, Tanemura M, Tanida T, Tomimaru Y, et al. Clinicopathological Significance of Leucine-Rich a2-Glycoprotein-1 in Sera of Patients With Pancreatic Cancer. Pancreas. 2015;44: 93-8.

15. Ji S, Qin Y, Liang C, Huang R, Shi S, Liu J, et al. FBW7 (F-box and WD repeat domain-containing 7) negatively regulates glucose metabolism by targeting the c-Myc/TXNIP (thioredoxin-binding protein) axis in pancreatic cancer. Clin Cancer Res. 2016;22:3950-6. 
16. Isidro RA, Isidro AA, Cruz ML, Hernandez S, Appleyard CB. Double immunofluorescent staining of rat macrophages in formalin-fixed paraffin-embedded tissue using two monoclonal mouse antibodies. Histochem Cell Biol. 2015;144:613-21.

17. O'Donnell LC, Druhan LJ, Avalos BR. Molecular characterization and expression analysis of leucinerich alpha2-glycoprotein, a novel marker of granulocytic differentiation. J Leukoc Biol. 2002;72:47885.

18. Serada S, Fujimoto M, Terabe F, lijima H, Shinzaki S, Matsuzaki S, et al. Serum leucine-rich alpha-2 glycoprotein is a disease activity biomarker in ulcerative colitis. Inflamm Bowel Dis. 2012;18:216979.

19. Shinzaki S, Matsuoka K, lijima H, Mizuno S, Serada S, Fujimoto M, et al. Leucine-rich Alpha-2 Glycoprotein is a Serum Biomarker of Mucosal Healing in Ulcerative Colitis. J Crohns Colitis. 2017;11:84-91.

20. Ha YJ, Kang EJ, Lee SW, Lee SK, Park YB, Song JS, et al. Usefulness of serum leucine-rich alpha-2 glycoprotein as a disease activity biomarker in patients with rheumatoid arthritis. J Korean Med Sci. 2014;29:1199-204.

21. Kentsis A, Ahmed S, Kurek K, Brennan E, Bradwin G, Steen H, et al. Detection and diagnostic value of urine leucine-rich a-2-glycoprotein in children with suspected acute appendicitis. Ann Emerg Med. 2012;60:78-83.e1.

22. Andersen JD, Boylan KL, Jemmerson R, Geller MA, Misemer B, Harrington KM, et al. Leucine-rich alpha-2-glycoprotein-1 is upregulated in sera and tumors of ovarian cancer patients. J Ovarian Res. 2010;3:21.

23. Ladd JJ, Busald T, Johnson MM, Zhang Q, Pitteri SJ, Wang H, et al. Increased plasma levels of the APC-interacting protein MAPRE1, LRG1, and IGFBP2 preceding a diagnosis of colorectal cancer in women. Cancer Prev Res (Phila). 2012;5:655-64.

24. Wang Y, Xing Q, Chen X, Wang J, Guan S, Chen X, et al. The Clinical Prognostic Value of LRG1 in Esophageal Squamous Cell Carcinoma. Curr Cancer Drug Targets. 2019;19:756-63.

25. Ramirez-Ardila DE, Ruigrok-Ritstier K, Helmijr JC, Look MP, van Laere S, Dirix L, et al. LRG1 mRNA expression in breast cancer associates with PIK3CA genotype and with aromatase inhibitor therapy outcome. Mol Oncol. 2016;10: 1363-73.

26. Zhong D, Zhao S, He G, Li J, Lang Y, Ye W, et al. Stable knockdown of LRG1 by RNA interference inhibits growth and promotes apoptosis of glioblastoma cells in vitro and in vivo. Tumour Biol. 2015;36:4271-8.

27. Wang X, Abraham S, McKenzie JAG, Jeffs N, Swire M, Tripathi VB, et al. LRG1 promotes angiogenesis by modulating endothelial TGF- $\beta$ signalling. Nature. 2013;499:306-11.

\section{Figures}




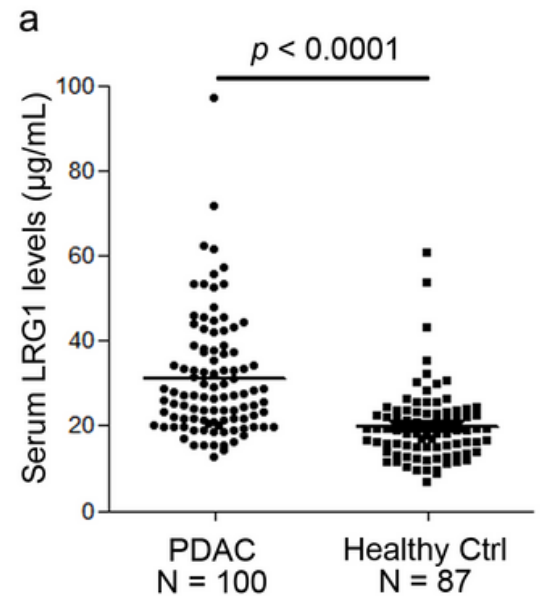

d

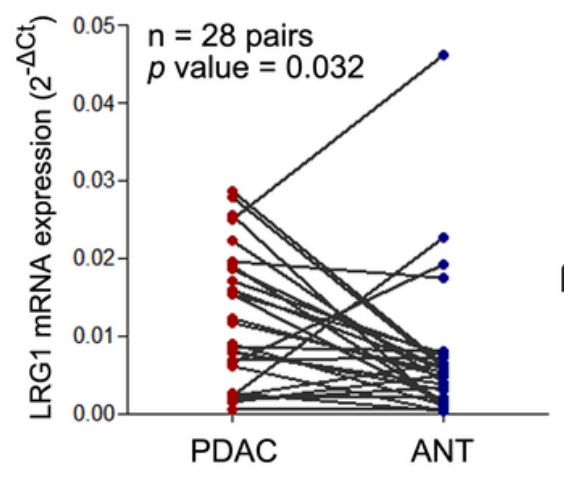

g

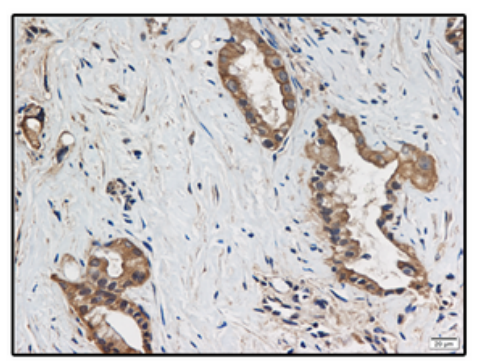

PDAC b

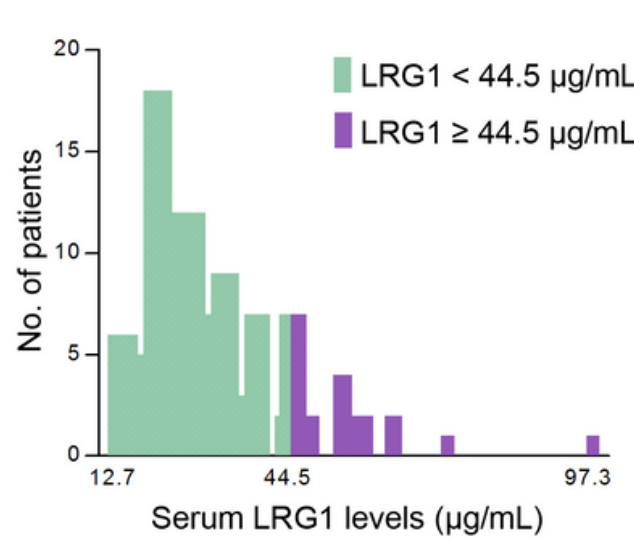

e

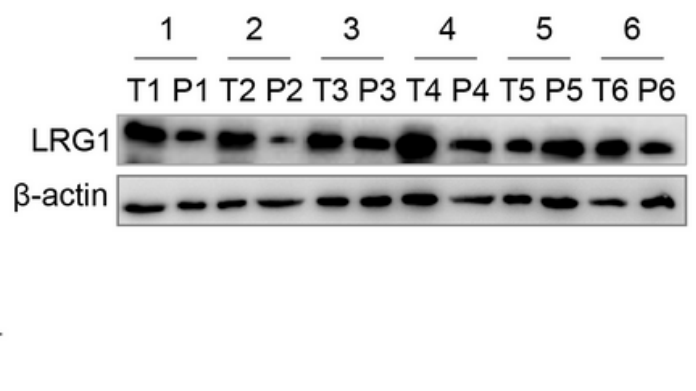

$\mathrm{h}$
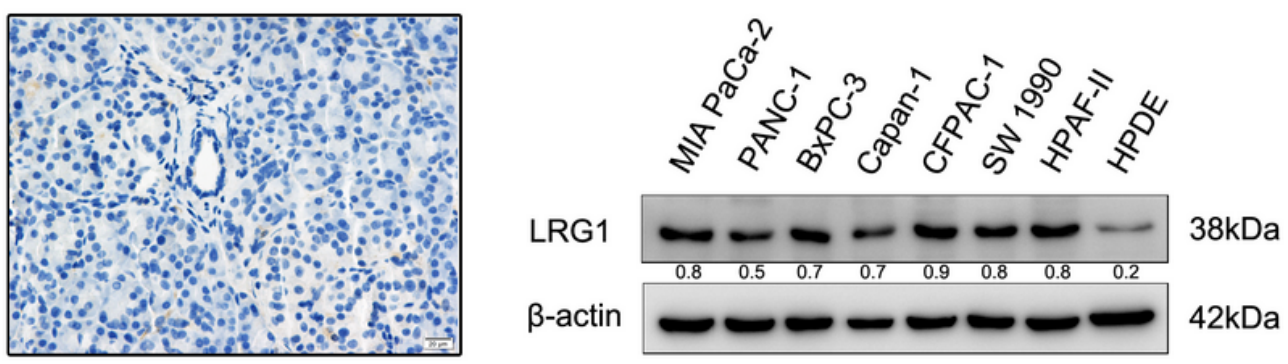

Figure 1

LRG1 expression in serums and tissues from patients with pancreatic cancer and cell lines. (a) Serum LRG1 levels in 100 patients with pancreatic cancer compared with those in 87 healthy controls. (b) Optimal cut-off for the serum LRG1 level for patients with pancreatic cancer determined with X-tile software. (c) Comparison of Kaplan-Meier survival curves by the cut-off. (d) Comparison of LRG1 mRNA expression among 28 paired cancer and adjacent normal tissues. (e) LRG1 protein expression among 6 paired cancer and normal tissues by western blot analysis. (f) Comparison of H-scores for LRG1 among 35 paired tissue samples by IHC analysis. (g) Representative images of IHC staining for LRG1 expression 
in pancreatic cancer and adjacent normal tissues (scale bar: $20 \mu \mathrm{m}$ ). (h) LRG1 protein expression in 7 pancreatic cancer cell lines and normal HPDE cells. ANT, adjacent normal tissue; Ctrl, control; PDAC, pancreatic ductal adenocarcinoma.

a
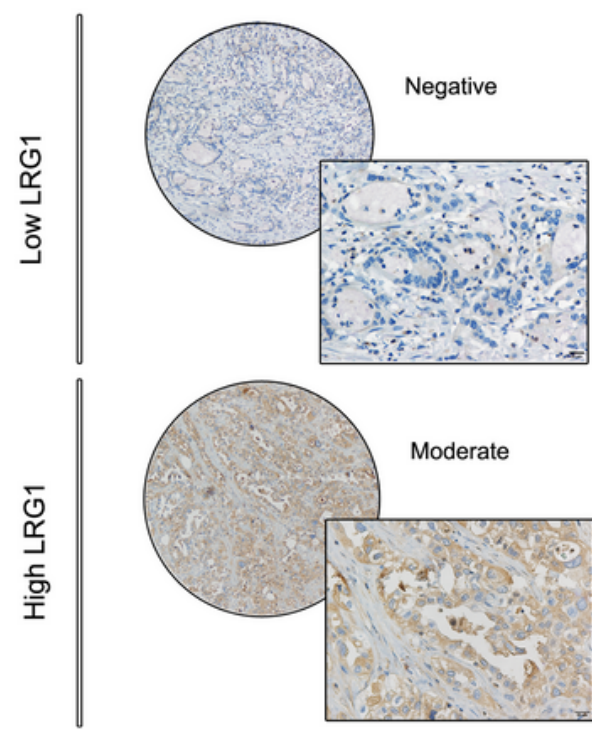

b

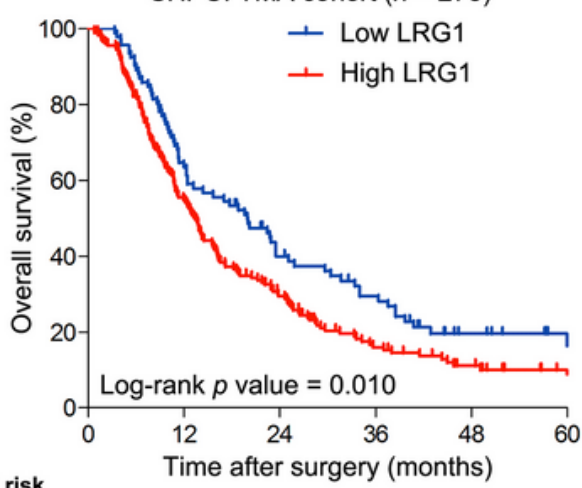

Moderate

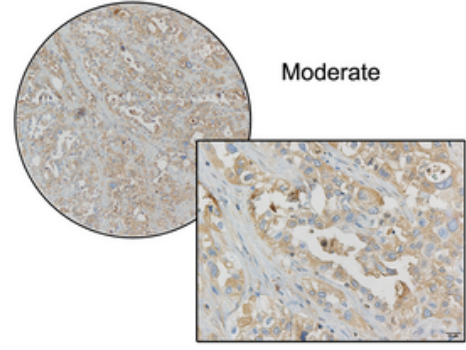

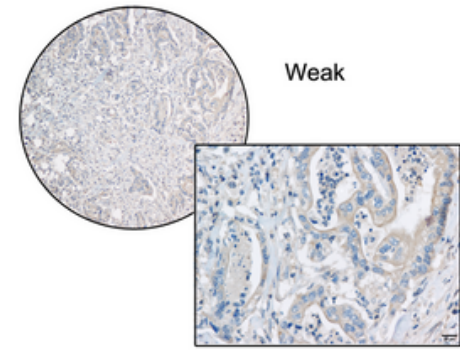

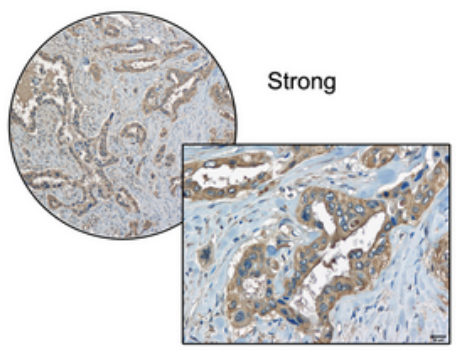

C

TCGA RNA-seq cohort $(n=176)$

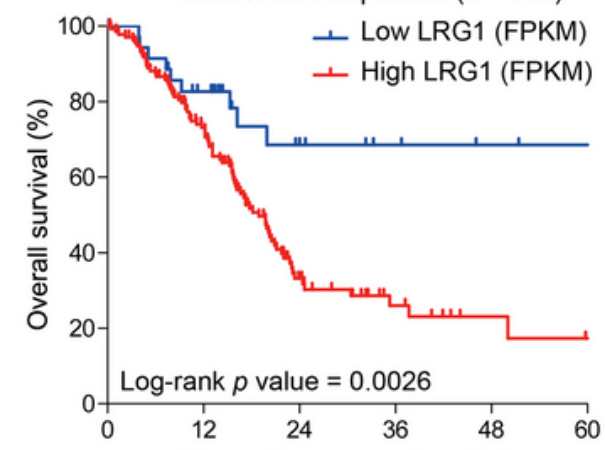

Time after surgery (months)

No. at risk

\begin{tabular}{|c|c|c|c|c|c|c|c|c|c|c|c|c|}
\hline Low LRG1 & 92 & 57 & 35 & 24 & 8 & 2 & Low LRG1 & 36 & 27 & 13 & 10 & 8 \\
\hline High LRG1 & 181 & 97 & 49 & 23 & 12 & 3 & High LRG1 & 140 & 88 & 25 & 11 & 5 \\
\hline
\end{tabular}

d

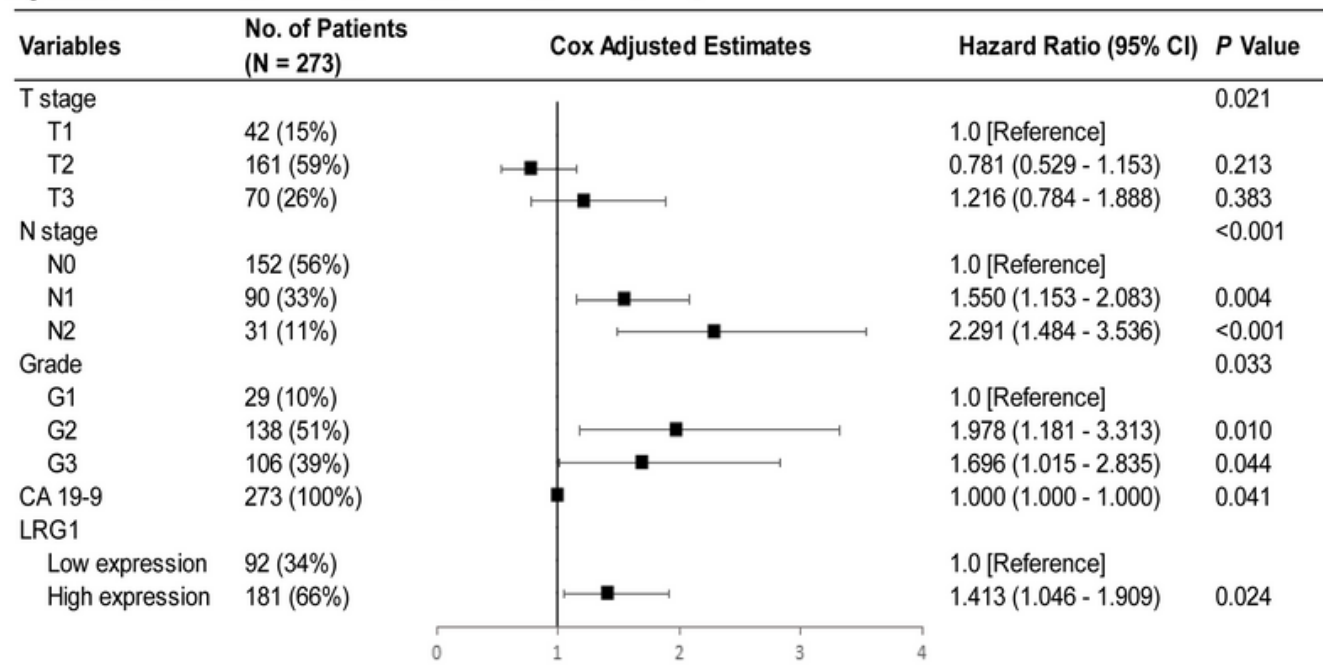

Figure 2

Prognostic value of LRG1 expression in pancreatic cancer. (a) Representative images of IHC staining for LRG1 expression in TMAs (scale bar: $20 \mu \mathrm{m}$ ). (b) Comparison of overall survival based on high and low 
LRG1 expression in 273 patients with pancreatic cancer from our institutional cohort. (c) Comparison of overall survival based on high and low LRG1 expression in 176 patients with pancreatic cancer from The Cancer Genome Atlas dataset. (d) Forest plot of LRG1 expression and the clinicopathological characteristics and overall survival of 273 patients with pancreatic cancer from our institutional cohort. FPKM, fragments per kilobase per million; SHPCl, Shanghai Pancreatic Cancer Institute; TCGA, The Cancer Genome Atlas; TMA, tissue microarray.

a

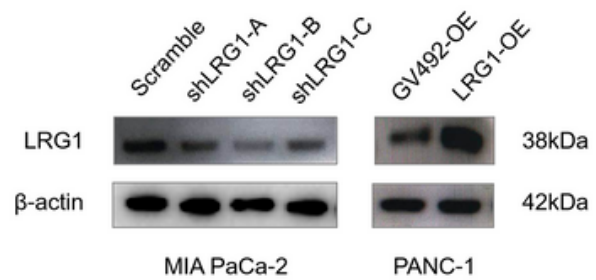

b

$\rightarrow$ MIA PaCa-2-Scramble
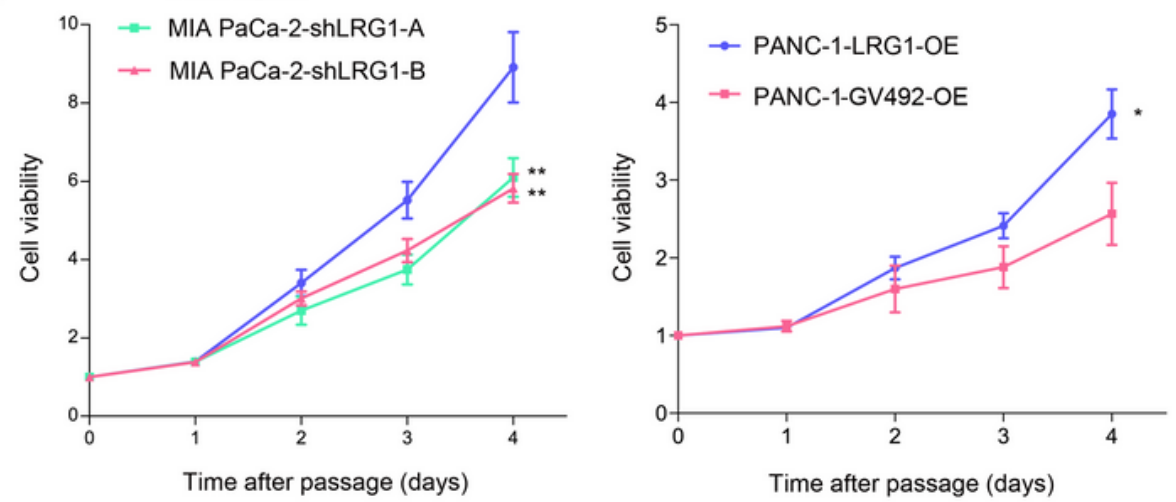

C
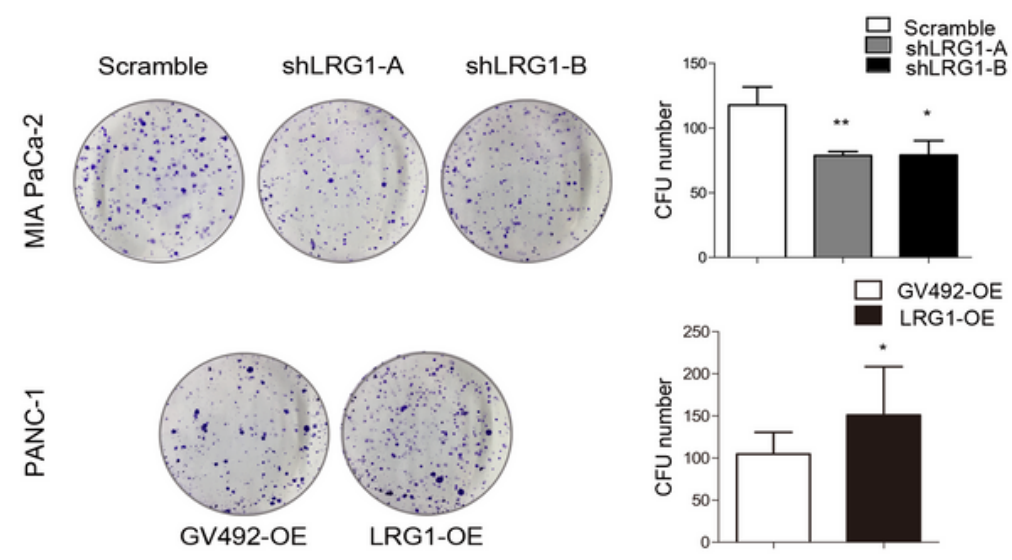

d

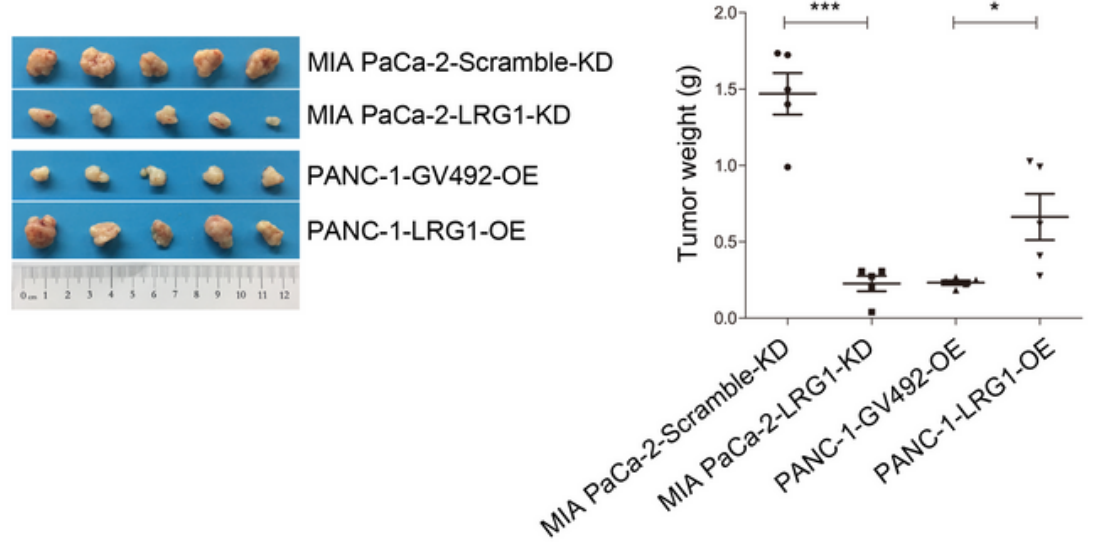

Figure 3 
Effect of LRG1 expression on the proliferation of pancreatic cancer cells. (a) Knockdown and overexpression of LRG1 in pancreatic cancer cells shown by western blot analysis. (b) CCK-8 assays in LRG1-knockdown or LRG1-overexpressing cells at 24, 48, 72, and $96 \mathrm{~h}$. (c) Colony formation assays in LRG1-knockdown or LRG1-overexpressing cells. (d) In vivo tumorigenicity assays using LRG1-knockdown or LRG1-overexpressing cells. ${ }^{*} \mathrm{P}<0.05 ;{ }^{* * \mathrm{P}}<0.01 ; * \star \star \mathrm{P}<0.001$.

a
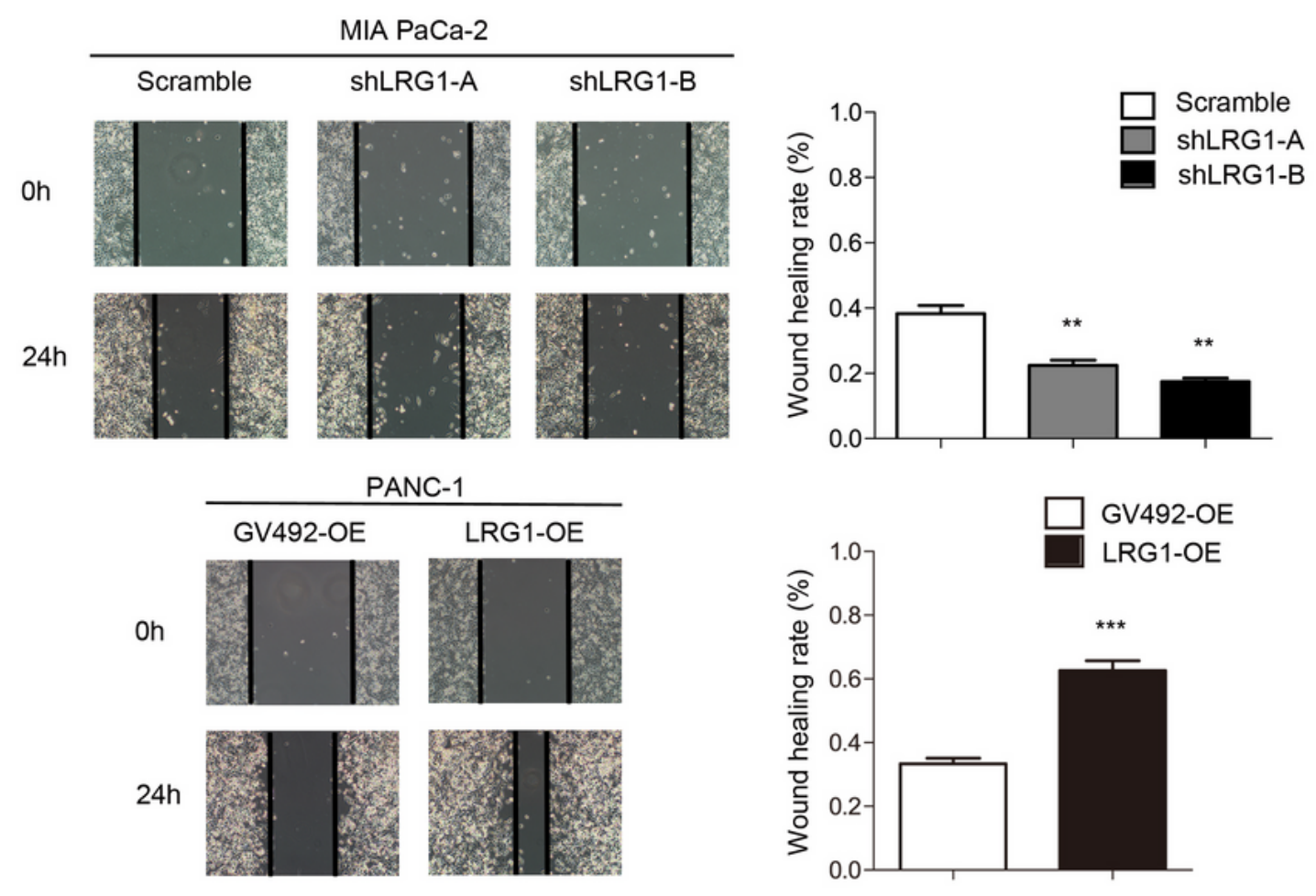

b
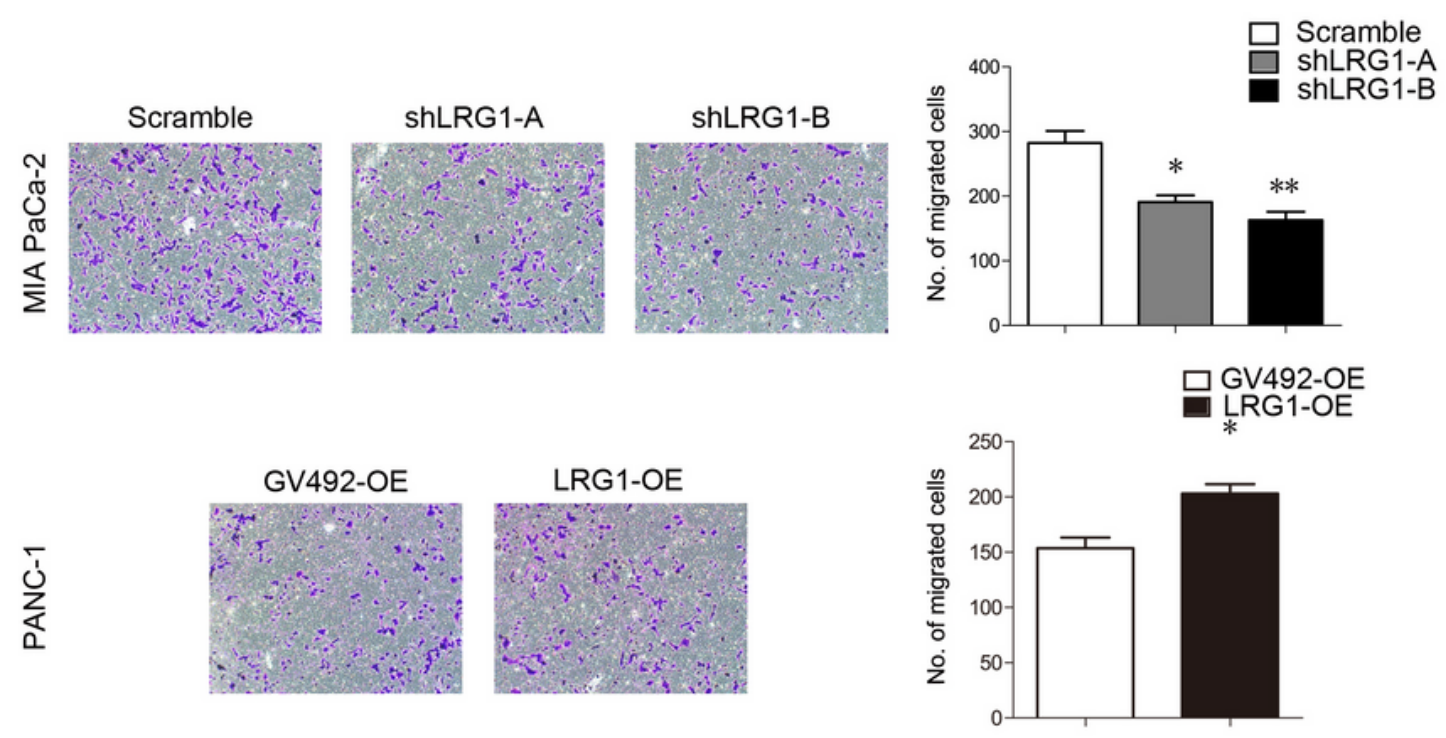

Figure 4 
Effect of LRG1 expression on the migration of pancreatic cancer cells. (a) Wound healing assays of LRG1-knockdown or LRG1-overexpressing cells. (b) Transwell migration assays of LRG1-knockdown or LRG1-overexpressing cells. ${ }^{*} \mathrm{P}<0.05 ;{ }^{* *} \mathrm{P}<0.01$; ${ }^{* *} \mathrm{P}<0.001$.

\section{a}

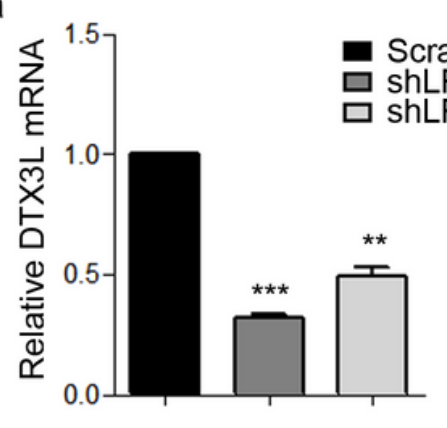

MIA PaCa-2

C

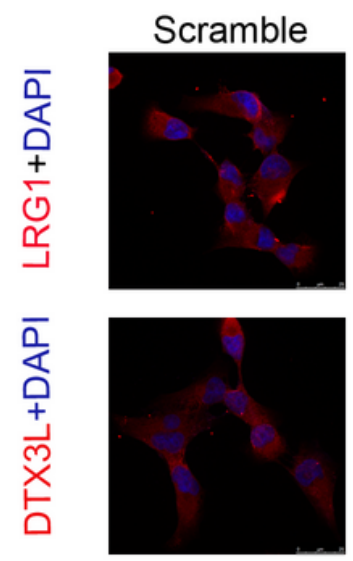

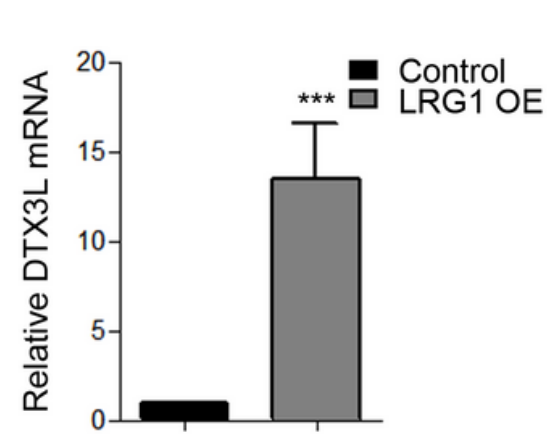

PANC-1 b

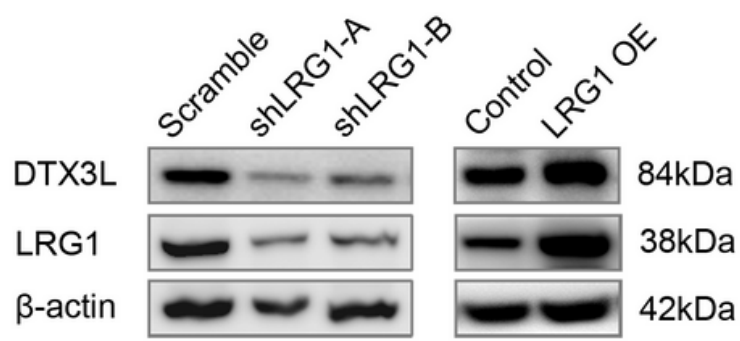
MIA PaCa-2

PANC-1

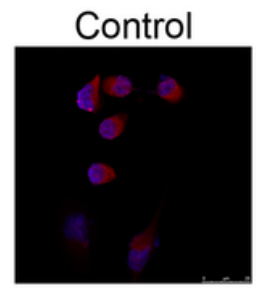

LRG1 OE
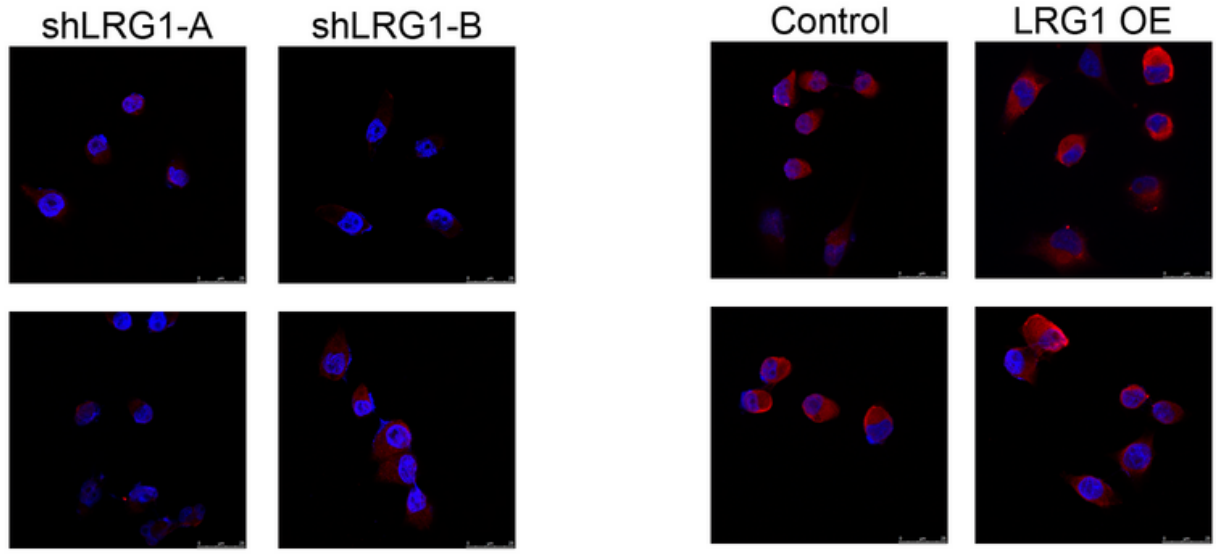

MIA PaCa-2

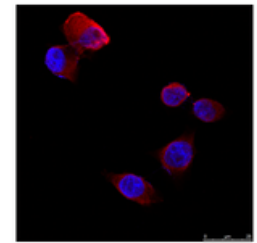

PANC-1

\section{Figure 5}

Validation that DTX3L is a downstream molecule of LRG1. (a) Alterations in the mRNA levels of DTX3L in pancreatic cancer cells after LRG1 knockdown or overexpression. (b) Alterations in the protein levels of DTX3L in pancreatic cancer cells after LRG1 knockdown or overexpression. (c) Expression of DTX3L after LRG1 knockdown or overexpression using immunofluorescence staining. 
a
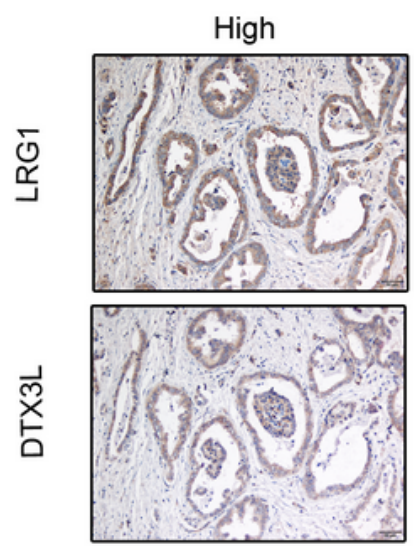

C

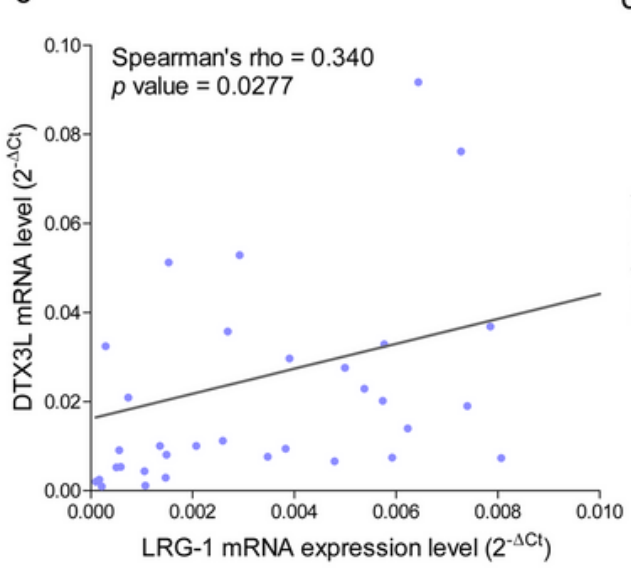

Low
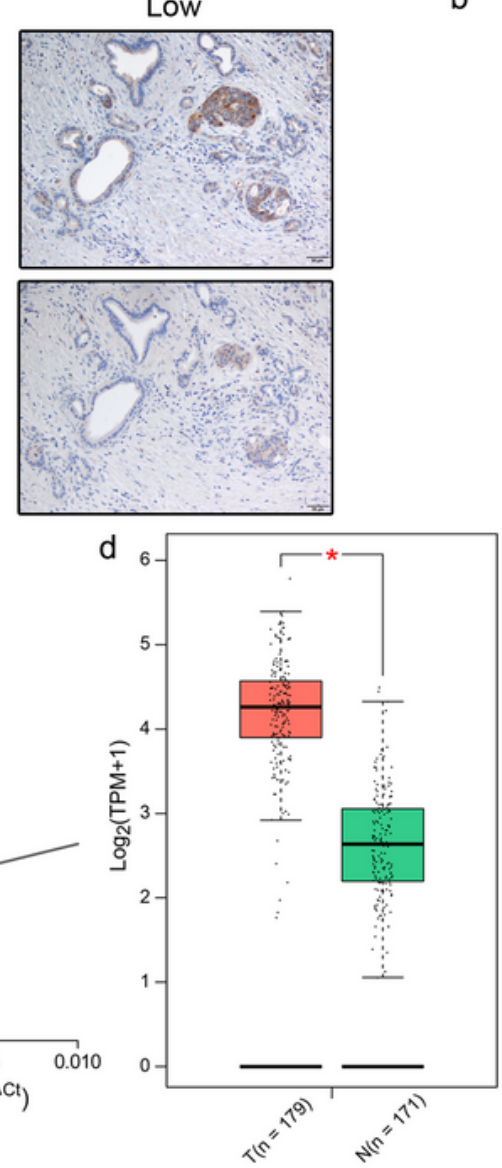

b
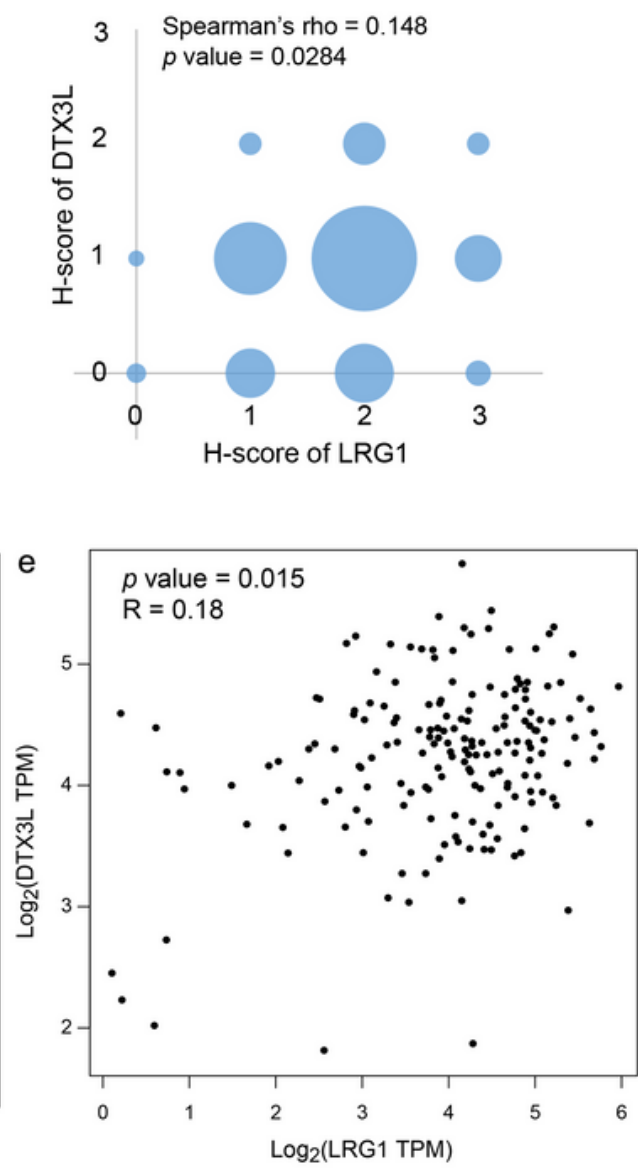

f

DAPI

LRG1
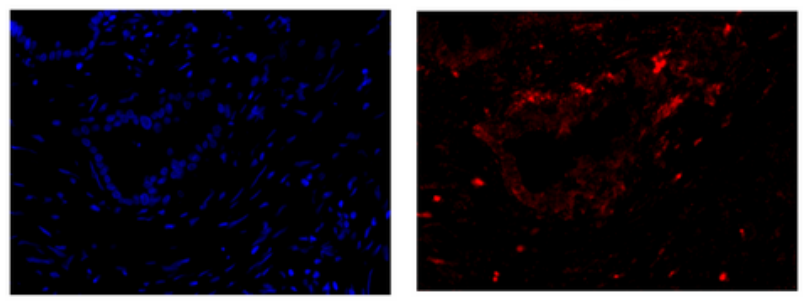

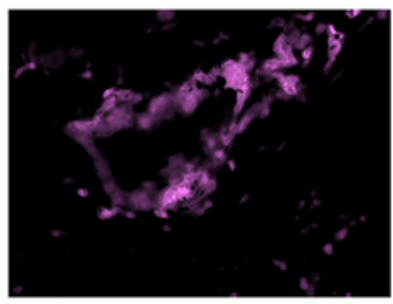

DTX3L

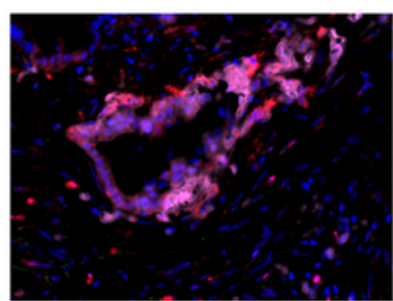

DAPI/LRG1/DTX3L

\section{Figure 6}

Correlation between LRG1 and DTX3L. (a) Representative images showing IHC staining for both LRG1 and DTX3L. (b) Bubble plot showing the correlation between LRG1 and DTX3L 273 patients with pancreatic cancer. (c) Correlation between the mRNA levels of LRG1 and DTX3L in 33 frozen pancreatic cancer tissues. (d) DTX3L expression in pancreatic cancer tissues $(n=179)$ and normal tissues $(n=171)$ from The Cancer Genome Atlas dataset (*P < 0.05). (e) Correlation between the mRNA expression levels 
of LRG1 and DTX3L in 179 patients with pancreatic cancer from The Cancer Genome Atlas dataset. (f) Double immunofluorescent staining showing the coexpression of LRG1 and DTX3L in cancerous epithelial cells. N, normal; T, tumor; TPM, transcripts per million. 